\title{
Automated Bottle Replenishment Plant using Programmable Logic Controller
}

\author{
P.K. Das ${ }^{1}$, V. Kumar ${ }^{2}$ and S.K. Singh ${ }^{3}$ \\ Student in Department of Applied Electronics \& Instrumentation, GIET, Gunupur, Odisha, India ${ }^{1,2,3}$
}

\begin{abstract}
A Programmable Logic Controller, PLC is a digital computer used for automation. It is an interface between program and the inputs. It is a programmable software. A PLC is an example of a real time application and therefore used to control various devices. The PLC works depending by the inputs given and their state, turning on/off its outputs. The user enters a program, usually through software which gives the results. PLC is used in many "real world" applications. For all application that needs some type of electrical signals, PLC works on the basis of inputs given by the user. Automation is used for all control systems and the technologies in PLC is use to reduce the human work and helps in increasing the production. PLC plays an important role in the world of automation industry . It acts a major function in the automation field which tends to reduce the complexity, increases safety and cost efficient. In this system we have applied a PLC based control system in an automatic bottle filling station.
\end{abstract}

Keywords: PLC, Bottle Replenishment, filling plant, Logixpro, SCADA, Bottle Capping, Automation, Damage detecting sensor.

\section{INTRODUCTION}

The aim of this project is to design PLC Based automatic bottle filling system that sense the presence of bottle and level of liquid in it and then fills it accordingly up to a fixed level. SCADA is interfaced with the PLC to see the live production and we can also see the counts of bottles on our computer screen using SCADA. We can also start-stop our project, change the value of counter from SCADA. - Filling is a task carried out by a machine that packages liquid products such as cold drinks or water. Traditional methods of bottle filling involved placing bottles onto a conveyor and filling only one bottle at a time. This method is time consuming and expensive. Our paper aims at filling and capping bottles simultaneously. The filling and capping operation takes place in a synchronized manner. It also includes a user-defined volume selection menu through which the user can input the desired volume to be filled in the bottles. The entire system is more flexible and time saving. The filling and capping operations are controlled using a Programmable Logic Controllers (PLC'S). This is because PLC's are very flexible, cost effective, space efficient and reduces complexity. By programming the PLC we control the entire system. SCADA (Supervisory Control and Data Acquisition) is used to monitor the process

\section{METHODOLOGY}

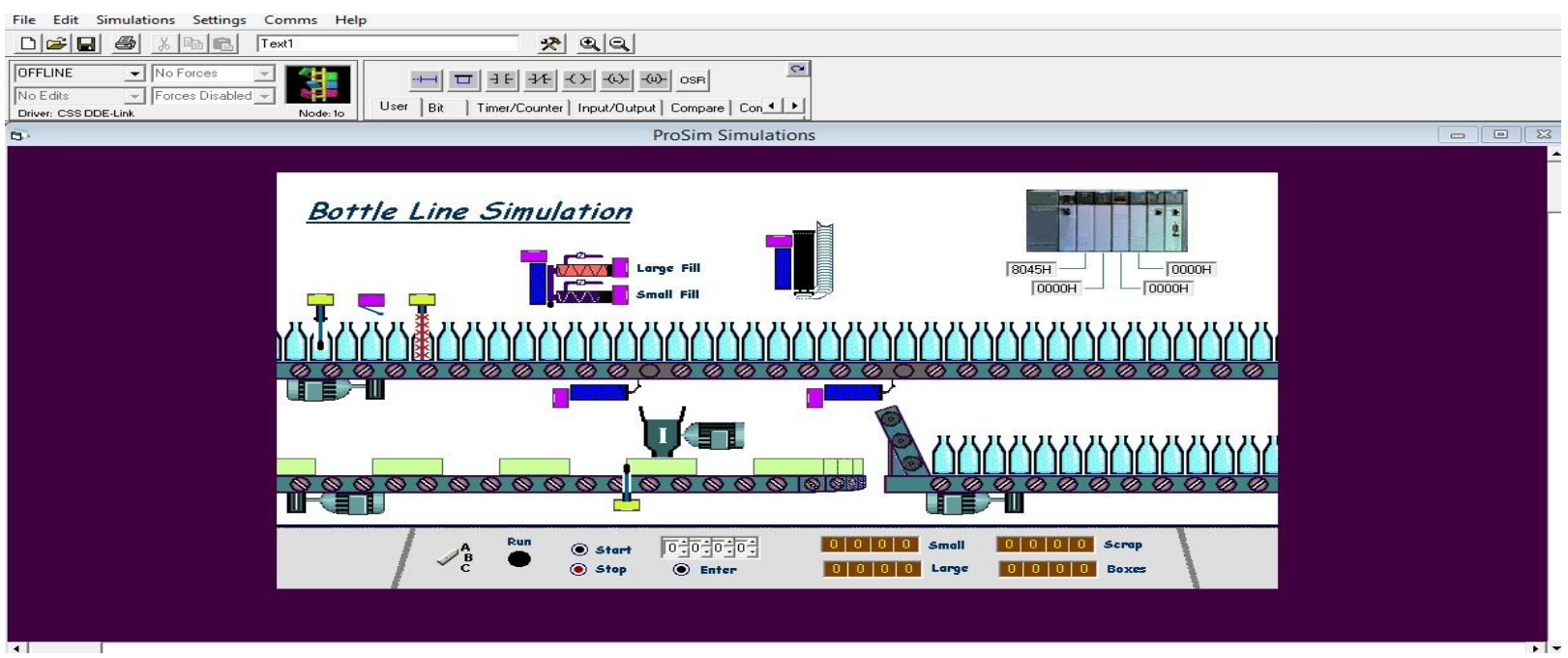

Figure1 : Bottle Line Simulator view 
Bottles are kept in position over a conveyor belt. An IR sensor is used to detect their presence. Next is a switch which will be in OFF condition when a large bottle will come then the switch will be in ON condition. Then there is a sensor which controls the quality of the bottles.Depending on the output of the switch the corresponding pumps switch on and filling operation takes place i.e. for large and small bottles. If the particular bottle is defective then the both pumps are off and the bottle is rejected and the bottle is thrown away from the main conveyor and pushed to the crusher situated below. Then the crusher will crush the bottle and put the crushed bottles in the boxes which is present in the second conveyor. Once the box is filled the level sensor is $\mathrm{ON}$ and the conveyor moves and next empty box came. Now the filled bottle is sealed by cap. Next is an sensor which detects the size of the bottle, if it large it will be bring down to the third conveyor and the small one will move forward on the same conveyor for further packaging.

\section{PROCESS DESCRIPTION}

This chapter gives a detailed explanation of the various processes taking place in a complete bottling system. The filling and capping operations take place in a sequential manner as shown in FIG II. When no bottles are kept in the input the system is reset. Depending on the number of bottles fed into the input side, the corresponding bottles are filled and capped. The process is also provided with a user defined volume selection menu.

\section{A. BLOCK DIAGRAM}

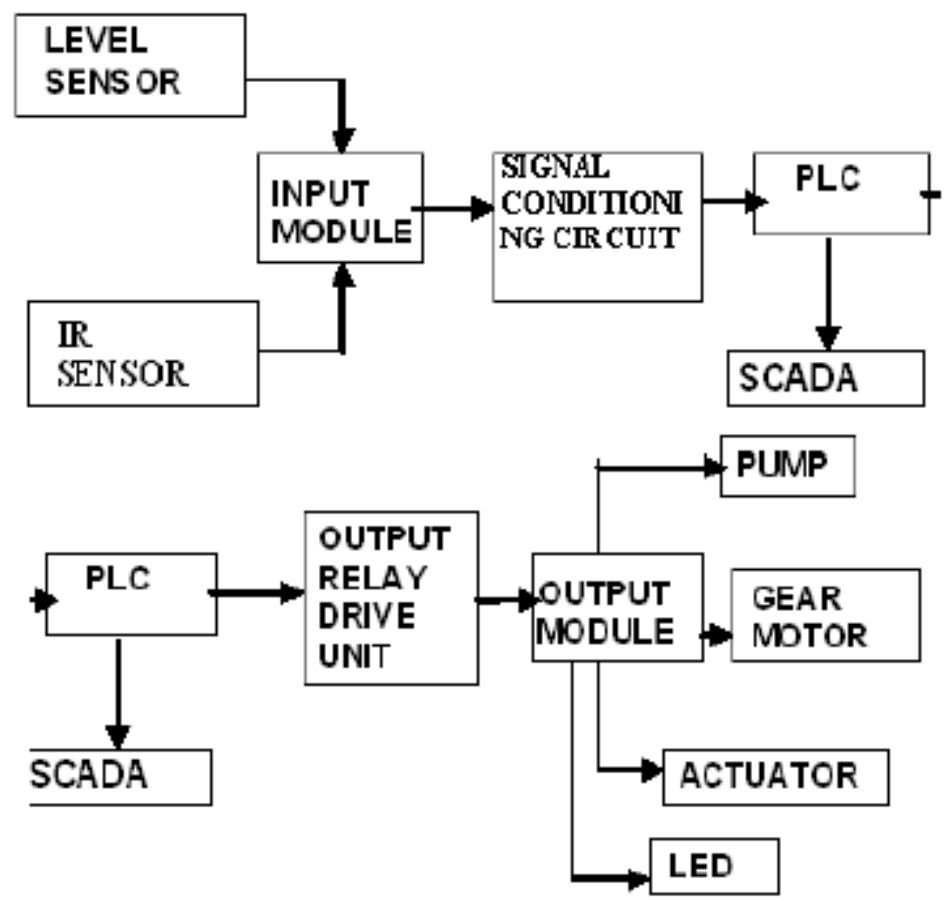

\section{B. INPUT MODULE}

The input module includes the IR sensors and level sensors. There are four pairs of IR sensors whose output is given as an input to the PLC. Three pairs of IR sensors which are used to detect the bottles at the input and one more is used to sense the bottles for filling and capping operations. The 3 IR pair sensors kept near the input side, where the bottles are fed into the conveyor, are called detection sensors and the one used for stopping the conveyor at the filling and capping operation side is called the stop sensor. There are seven levels sensors used. Two level sensors are used in tank 1 (concentrate tank) to denote the high level and low level and similarly two sensors are used in tank 2 (tank containing water). Three level sensors are used in the process tank to denote the low, middle and high levels. Thus, these are the inputs given to the input module.

\section{SIGNAL CONDITIONING}

The output of the sensors cannot be given directly to the PLC as the input voltage to the PLC should be $24 \mathrm{~V}$. Hence they are given through signal conditioning circuits which condition the input signals and in turn give it as an input to the PLC. For safety purposes the inputs are not given directly to the PLC. They are given through relay circuits. The relay consist of 3 terminals - common, NO and NC. The $24 \mathrm{~V}$ which is to be inputted into the PLC will be available in 


\section{ISO 3297:2007 Certified}

Vol. 5, Issue 4, April 2017

the common terminal. Hence depending upon the necessary signals, the circuit closes or opens thereby connecting to the PLC.

\section{PLC}

PLC is a solid state device. They are well-adapted to a range of automation tasks .All control operations (filling and capping) are done using the PLC. The entire bottling process is automated by feeding the necessary conditions into the PLC using ladder logic. Ladder logic is one of the methods of programming a PLC. Thus, depending on the logic developed the various operations take place and the filling and capping of bottles are done. PLC consists of an I/O unit, central processing unit, and a memory unit. The input/output unit of the PLC acts as an interface to the real world. Inputs from real work are given to the input unit which is manipulated based on the programming, and the results are given back to the real world through the output unit of the PLC. All logic and control operations, data transfer and data manipulation operations are done by the central processing unit. The results and statuses are stored in the memory of the PLC. PLC's are used for a wide range of applications especially in the field of control and automation.

\section{E. OUTPUT RELAY DRIVE UNIT}

The operating voltage of the output devices is low when compared to that of the PLC. The output of the PLC is 24V whereas output devices such as gear motors and pump require only $12 \mathrm{~V}$ for their operation. Hence the output signals from the PLC are given through a relay drive unit which drives the output devices by supplying the optimum voltage required for their operation. The $12 \mathrm{~V}$ required for their operation is available at the common terminal of the relay unit.

\section{F. OUTPUT MODULE}

The various output devices used in the bottling process are gear motors, pumps, actuators and LED's. These are connected to the output module. The gear motor is used to run the conveyor in forward direction. There are totally five pumps used for the filling process. Three pumps are connected to the process tanks and the other two pumps are connected to the concentrate (tank 1) and water tank (tank 2) respectively. Actuators are used for capping the bottles. The actuator applies a force on the caps which in turn corks the bottles tightly. These are the various output devices used in the bottling process. The basic process (as shown in fig I ) involved in the bottling and capping is explained as follows

\section{SERIES OF OPERATIONS}

\section{A. BOTTLE DETECTION USING SENSORS}

Bottles are kept in position in their respective holders which are fixed to the conveyor at the input side. IR sensors are used to detect the presence of bottles in the holder. Depending on the output of the sensors the filling and capping operation takes place. A time delay is given in order to set the status of the bottles. If bottle 1 is present the corresponding status bit in PLC is set to 1 else it is set to 0 . The outputs of these sensors are given to the PLC and depending on this output the filling and capping process for the bottles takes place. Thus if all the $\mathrm{n}$ bottles are present in the input side then the sensor gives the corresponding output to the PLC which in turn switches ON the corresponding pumps for filling operation to take place. If a particular bottle is not present the corresponding pumps remain OFF.

\section{B. FILLING OPERATION}

Once the bottles are detected in the input side the conveyor motor switches ON and it starts moving in the forward direction. The bottles then reach the desired position for filling and the conveyor stops. The corresponding pumps in process tank switch ON and filling operation takes place. For e.g. if only bottle 1 is present then pump 1 switches ON and pump 2 and pump 3 remain switched OFF. There are three tanks present in the filling side namely: process tank, concentrate tank (tank 1) and tank to store water (tank 2).Tank 1 and tank 2 have low level and high level sensors (LLS and HLS) respectively. Process tank has three level sensors (LLS, HLS and MLS).MLS is used to denote the middle level of the tank. When the liquid in the process tank reaches below low level (LLS) pumps in tank 1 and tank 2 switches on and the process tanks get filled. When the level of liquid reaches high level (HLS) the pumps in tank 1 and 2 switch off.

\section{USER-DEFINED VOLUME}

The filling operation is accompanied with a user defined volume selection menu. The desired volume is fed into the PLC and depending on the volume the filling of liquid takes place. The filling is done using timing operations. Thus the pump remains on for the preset value of the timer and switches off once time is out. Once the filling process is done the conveyor starts moving again. 


\section{ISO 3297:2007 Certified}

Vol. 5, Issue 4, April 2017

\section{CAPPING OPERATION}

The bottles are transported to the capping arrangement. IR sensors are kept to stop the bottles in the desired position for capping to take place. Once the bottles reach the position the conveyor motor switches OFF. The capping of bottles is done using actuator arrangement. Three actuators which move in forward and reverse directions are used to cap the bottles. The actuator applies a force on the caps which are placed in their respective holders. This force helps the caps to fit into the bottles at the required position. Similar to filling, if a particular bottle is not present it does not get capped. Thus the capping is done and the conveyor starts moving again. When the capping operation for one batch is done simultaneously the filling operation for another batch takes place. The entire sequence of operations continues until there are not bottles present in the input side. All these are automatically coordinated using the PLC. PLC gets the input from the input devices and sends the signals to the various output devices depending on the conditions that are fed into the PLC. Thus bottles get filled and capped.

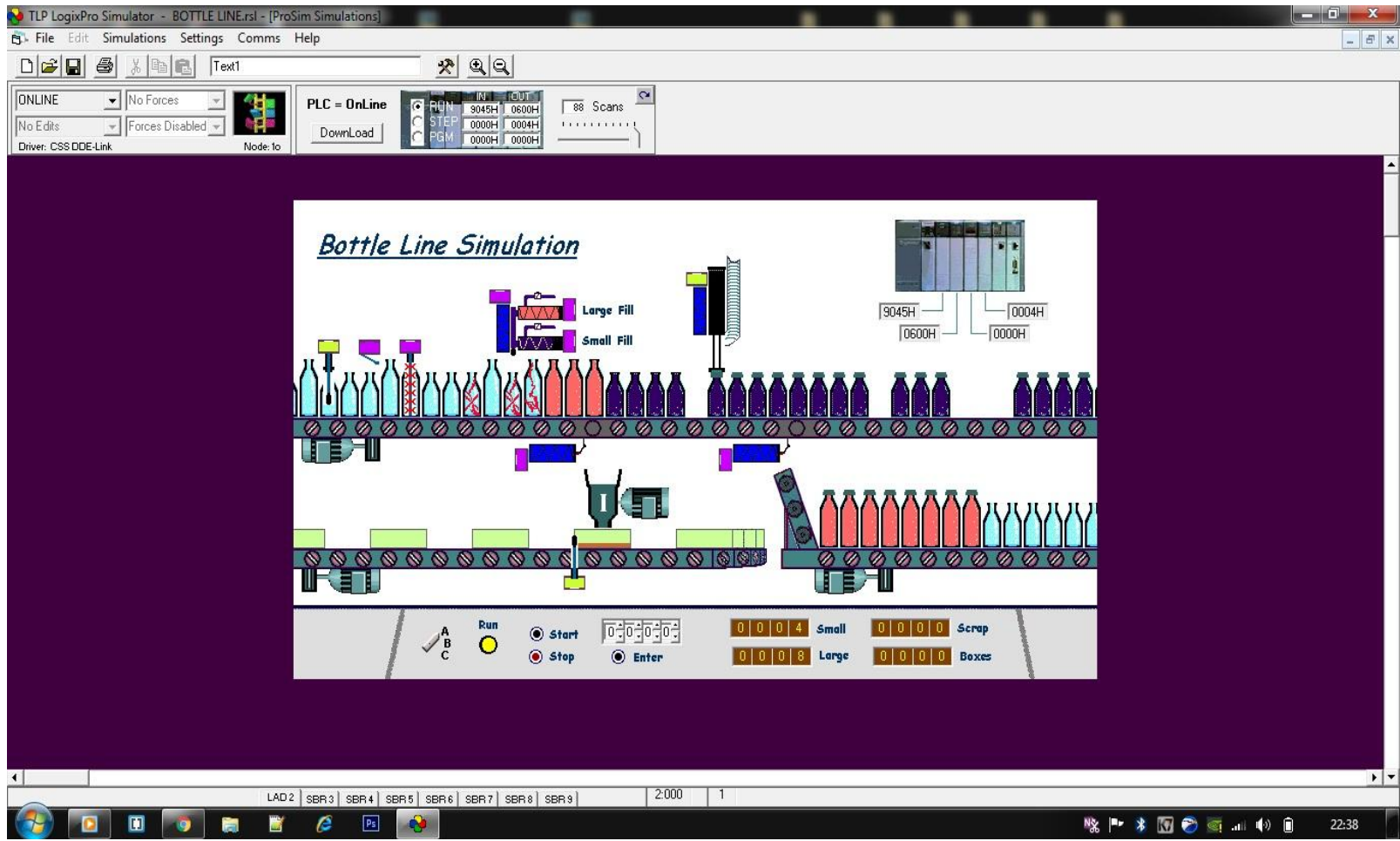

Figure 2- Visualization of Bottle Replenishment Plant

\section{CONCLUSION}

The main objective of this paper was to develop a bottle filling and capping system based on certain specifications. This was successfully implemented. We consider this paper as a journey where we acquired knowledge and also gained some insights into the subject which we have shared in this report. A lot of additional features like user defined volume specification etc. were added in the different stages in our work and the desired results were obtained. PLC was used to control the various operations and monitoring was done using SCADA. More features can be added to this system as follows: depending on the size, shape and weight of the bottles, filling and capping operations can be implemented. Capping operation can be improvised using a piston arrangement.

\section{REFERENCES}

[1] Shaukat.N, ,PLC based automatic liquid filling process, Multi Topic Conference 2002,IEEE Publications.

[2] Dunning Gray (1998) - 'Introduction to Programmable Logic Controllers' - Delmar publishers, pp.421-428.

[3] Petruzella, Frank D.(2010)-'Programmable logic Controllers' - Tata McGraw Hill Education,pp.-612.

[4] Rashid, M.H. (2010) - 'Power Electronics' - British Library of Congress

[5] Rehg,James A. Glenn J Sartori (2009) - 'Programmable Logic Controllers'- Pearson prentice Hall, pp. $230-239$.

[6] Hemant Ahuja, Arikha Singh, Saubhagya Tandon, Shreya shrivastav, Sandeep Patil, "Automatic Filling Management System For Industries", IJETAE, Vol. 4, Special Issue 1, February - 2014

[7] Nabil Shaukat, "A PLC Based Automatic Liquid Filling Process", IEEE, publication page No. 226-233, 2002. 


\section{IJIREEICE

[8] Ahmed ulla Abu Saeed, Md. Al-mamun, A.H.M. Zadidul Karim, “ Industrial Application Of PLCs in Bangladesh”, IJSER, Vol. 3, Issue 6, June 2012

\section{BIOGRAPHIES}

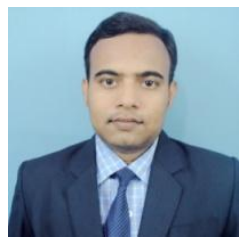

Mr. Prabir Kumar Das is pursuing his bachelor in Applied Electronics \& Instrumentation Engineering from GIET, Gunupur, Odisha, India. His interest field of research is Automation and Embedded System

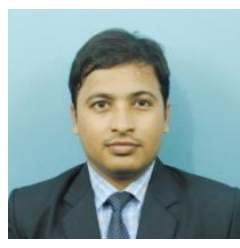

Mr. Vishal Kumar is pursuing his bachelor in Applied Electronics \& Instrumentation Engineering from GIET, Gunupur, Odisha, India. His interest field of research is Automation and Embedded System

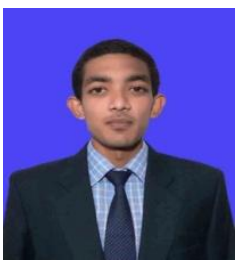

Mr. Shubham Kumar Singh is pursuing his bachelor in Applied Electronics \& Instrumentation Engineering from GIET, Gunupur, Odisha, India. His interest field of research is Automation and Embedded System 